\title{
cDNA Cloning of a Sorghum Pathogenesis-Related Protein (PR-10) and Differential Expression of Defense- Related Genes Following Inoculation with Cochliobolus heterostrophus or Colletotrichum sublineolum
}

\author{
Sze-Chung Clive Lo, John D. Hipskind, and Ralph L. Nicholson \\ Department of Botany and Plant Pathology, Purdue University, West Lafayette, IN 47907-1155, U.S.A. \\ Accepted 9 February 1999.
}

\begin{abstract}
A sorghum cDNA clone was isolated by differential screening of a cDNA library prepared from mesocotyls (cultivar DK18) inoculated with fungal pathogens. The deduced translation product shows sequence similarity to a family of intracellular pathogenesis-related proteins (PR-10) with a potential ribonuclease function. We studied the accumulation of PR-10 and chalcone synthase (CHS) transcripts in mesocotyls following inoculation with Cochliobolus heterostrophus or Colletotrichum sublineolum. CHS is involved in phytoalexin synthesis in sorghum. Coordinate expression of PR-10 and CHS genes was localized in the area of inoculation along with the accumulation of phytoalexins. $C$. heterostrophus is a nonpathogen of sorghum and cytological studies indicated that cultivar DK18 is resistant to $C$. $s u b$ lineolum, a sorghum pathogen. We demonstrated that the two fungi triggered different time courses of plant defense reactions. Inoculation with $C$. heterostrophus resulted in rapid accumulation of PR-10 and CHS transcripts after appressoria had become mature. Accumulation of these transcripts was delayed in plants inoculated with $C$. sublineolum until penetration of host tissue had been completed and infection vesicles had formed. Results suggest that different recognition events are involved in the expression of resistance to the two fungi used or that $C$. sublineolum suppresses the nonspecific induction of defense responses.
\end{abstract}

Additional keywords: anthracnose, Bipolaris maydis, 3-deoxyanthocyanidin, incompatible interactions.

Plants are naturally resistant to most potentially pathogenic microorganisms and are considered to express nonhost resistance to nonpathogens. Pathogens that are able to infect their host plants have developed ways to suppress or negate the defense mechanisms associated with nonhost resistance (Heath 1981; Johal et al. 1995). Host-pathogen incompatibility is the result of specific interactions that can often be defined by simple

Corresponding author: R. L. Nicholson

E-mail: nicholson@btny.purdue.edu

Current address of J. D. Hipskind: The Samuel Roberts Noble Foundation, Box 2180, 2510 Sam Noble Parkway, Ardmore, OK 73402, U.S.A.

Nucleotide and/or amino acid sequence data have been submitted to GenBank as accession number U60764. genetic relationships (gene-for-gene hypothesis) in many systems (Keen 1992). Recognition of potential pathogens by plants often evokes an array of defense-related responses at the site of attempted invasion. Production of pathogenesis-related (PR) proteins represents a component of the active plant defense repertoire. PR proteins are defined as plant proteins that are induced in pathological or related situations (van Loon et al. 1994), and were first described and characterized in tobacco leaves following infection by tobacco mosaic virus (van Loon and van Kammen 1970). Subsequently, based on serological and molecular properties, five families of closely related PR proteins (PR-1 to PR-5) have been found in most plants studied (van Loon 1985). According to the nomenclature suggested by van Loon et al. (1994), an additional six families (PR-6 to PR-11) have been included when the definition of PR proteins was extended to all novel proteins, with or without a known function, that are produced in plants in response to challenge by pathogens. Several of the PR proteins are hydrolytic enzymes, for example, chitinases (Collinge et al. 1993) and 1,3- $\beta$-glucanases (Kauffmann et al. 1987), which exhibit antimicrobial properties through degradation of cell wall components of bacterial and fungal pathogens. Some PR proteins appear to be antifungal because of their ability to bind to chitin (Ponstein et al. 1994), their structural sequence similarity to osmotin (Cutt and Klessig 1992; Stintzi et al. 1993), their proteinase-inhibitor-like properties (Roby and Esquerre-Tugaye 1987; Pautot et al. 1991), or their thionin-like characteristics (Bohlmann et al. 1988).

In contrast to most PR proteins, which are extracellular, intracellular PR proteins were first described in cultured parsley cells upon elicitor treatment (Somssich et al. 1988). These intracellular proteins have molecular masses and acidic characteristics similar to those of PR-1 proteins, but are not structurally related to the PR-1 proteins of tobacco. Parsley "PR1" has been suggested to represent the "type member" for a ubiquitous class of intracellular, defense-related proteins, the PR-10 family (van Loon et al. 1994). PR-10 proteins have been discovered in a number of species throughout the plant kingdom, including the dicots pea (Fristensky et al. 1988), bean (Walter et al. 1990, 1996), soybean (Crowell et al. 1992), alfalfa (Breda et al. 1996), and potato (Matton and Brisson 1989), and the monocot asparagus (Warner et al. 1992). A relationship between the expression of a PR-10 protein and disease resistance has been demonstrated in pea (Riggleman et al. 1985). The biological functions of PR10 proteins were speculated to be related to a possible ribonu- 
clease activity after significant amino acid sequence homology had been reported between a ginseng ribonuclease and parsley PR-10 proteins (Moiseyev et al. 1994). Interestingly, PR-10 proteins also share amino acid sequence similarity to pollen allergens of trees (Breiteneder et al. 1989) and to the major food allergen of celery (Breiteneder et al. 1995).

Sorghum mesocotyls inoculated with fungal pathogens have been used as a system for physiological and molecular studies of 3-deoxyanthocyanidin phytoalexin synthesis (Hipskind et al. 1996a; Lo and Nicholson 1998). The phytoalexin response is preceded by transcriptional activation of genes encoding the branch point enzymes in the phenylpropanoid pathway, including phenylalanine ammonia-lyase and chalcone synthase (CHS). 3-Deoxyanthocyanidins are structurally similar to anthocyanidins but their routes of synthesis have been shown to occur via separate pathways in sorghum (Hipskind et al. 1996a). In a recent study, we demonstrated that the phytoalexins are synthesized at the expense of light-induced anthocyanin accumulation in order to meet the immediate physiological and biochemical needs of the plant following fungal inoculation (Lo and Nicholson 1998). Differential screening of cDNA libraries derived from mRNA isolated from inoculated or elicitor-treated plants and cultured cells has been employed extensively for the identification of defense-related genes in many systems. We previously isolated a cDNA encoding a novel, leucine-rich repeat motif from inoculated sorghum mesocotyls (Hipskind et al. 1996b). This cDNA may represent a gene that is a component in signal perception and subsequent activation of defense-related genes in sorghum.

Following a similar procedure, we have now isolated and characterized another defense-related cDNA clone and found that the predicted amino acid sequence was similar to that in members of the PR-10 protein family. In addition, we also investigated the temporal expression of PR-10 and CHS genes in two different incompatible interactions. Sorghum seedlings were inoculated either with Cochliobolus heterostrophus, a nonpathogen of sorghum, or Colletotrichum sublineolum, the causal agent of the anthracnose disease of sorghum. To correlate the accumulation of mRNA with the fungal infection process, we also examined the two different interactions at the cytological level. Results demonstrated that the two fungal pathogens triggered differential temporal expression of plant defense reactions, which were correlated to their respective morphological development on the plant.

\section{RESULTS}

\section{Isolation of sorghum PR-10 cDNA.}

In an attempt to identify defense-related genes in sorghum, a cDNA library was constructed from mRNA isolated from mesocotyls (cv. DK18) $24 \mathrm{~h}$ after inoculation with C. sublineolum. cDNA clones corresponding to pathogen-induced transcripts were isolated from the library by a differential hybridization procedure. One of these clones was found to harbor an insert of $907 \mathrm{bp}$, which was fully sequenced and shown to contain a single open reading frame (ORF) spanning $483 \mathrm{bp}$, flanked by stretches of 69 and $352 \mathrm{bp}$ at the $5^{\prime}$ and $3^{\prime}$ ends, respectively (Fig. 1). Three potential translational stop codons and one putative polyadenylation site were recognized in the $3^{\prime}$ flanking region, which were followed by a short poly (A) tail. The ORF encoded a predicted polypeptide of 161 amino acid residues.
A basic BLAST search of on-line protein data bases (National Center for Biotechnology Information) revealed significant homologies between the sequences of the deduced translation product and members of the PR-10 protein family (Fig. 2): an asparagus wound-inducible protein, AoPR1 (49.7\% identity; Warner et al. 1992), a potato PR protein, pSTH-21 (31.8\% identity; Matton and Brisson 1989), a parsley PR protein, PR1-1 (31.6\% identity; Somssich et al. 1988), a pea disease resistance protein, pI49 (31.0\% identity; Fristensky et al. 1988), and a bean PR protein, PR-10a (29.0\% identity; Walter et al. 1996). PR-10 proteins are also structurally related to some tree-pollen allergens as well as two ribonucleases from ginseng. For example, the sequence of sorghum PR10 protein shows $33.3 \%$ identity to the birch tree-pollen allergen, BetvI (Breiteneder et al. 1989), 29.2\% identity to the celery major allergen, Api g 1 (Breiteneder et al. 1995), and $31.1 \%$ identity to ginseng ribonuclease 2 (Moiseyev et al. 1997). There are 10 strictly conserved amino acid residues in all the PR-10 and related proteins examined (Fig. 2). Most of them are Gly $(\mathrm{G})$ and Pro (P), which are probably essential for the polypeptide fold (Gajhede et al. 1996). Glu (E) -99, Glu (E) -150 , and Tyr (Y) -152 (Fig. 2) are the only conserved residues with functional groups that may be involved in the catalytic region (Moiseyev et al. 1997).

\section{Genomic Southern analysis.}

For genomic Southern analysis total DNA isolated from sorghum DK18 mesocotyls was digested to completion with EcoRI, HindIII, or XbaI. The hybridization probe was derived from the PR-10 cDNA clone, which shows no recognition sites for these restriction enzymes. Figure 3 reveals a number of signals of varying intensities and sizes in the different digested DNA samples. The hybridization pattern suggests there may be 6 to 8 copies of PR-10 genes in sorghum. One of the characteristics of PR-10 genes is that "they are almost invariably part of gene families" (Breda et al. 1996). However, the presence of multiple bands observed in Figure 3 could also be attributable to introns and/or allelic differences at a single locus.

\section{Expression of PR-10 and CHS genes in inoculated mesocotyls.}

To study the expression of PR-10 and CHS genes in response to fungal inoculation, Northern (RNA) blot hybridizations were performed with total RNA extracted from sorghum mesocotyls at various times after inoculation. PR-10 transcripts were first detected in tissues $4 \mathrm{~h}$ after inoculation with C. heterostrophus, a nonpathogen of sorghum. Accumulation of PR-10 transcripts reached a maximum level at $12 \mathrm{~h}$ after inoculation and then declined progressively (Fig. 4A). The estimated transcript size is about $0.9 \mathrm{~kb}$, which correlates with the length of the isolated cDNA clone. Since there may be a gene family of PR-10 proteins (Fig. 3), the pattern of transcript accumulation shown by Northern analysis may be the result of expression of one or more PR-10 genes in sorghum. CHS transcripts $(1.35 \mathrm{~kb})$ were first detected $6 \mathrm{~h}$ after inoculation with the same fungus and accumulation thereafter followed a pattern similar to that of the PR-10 transcripts (Fig. 4A). CHS is a key branch-point enzyme in the phenylpropanoid pathway, and its enzymatic activity and gene expression are closely related to phytoalexin biosynthesis in sorghum (Lue et al. 1989; Weiergang et al. 1996b). 
After inoculation of sorghum mesocotyls with the anthracnose pathogen $C$. sublineolum, PR-10 transcripts were not detected until $36 \mathrm{~h}$ after inoculation (Fig. 4B). Subsequently, there was a significant and sustained level of transcript accumulation throughout the course of the experiments (96 h after inoculation). Similarly, a detectable amount of CHS transcripts was first observed within $36 \mathrm{~h}$ of inoculation with the same pathogen (Fig. 4B). A substantially high level of CHS transcript accumulation was observed with a pattern similar to that of the PR-10 transcript. Thus, the results demonstrated that PR-10 and CHS genes were activated differently by the two different fungi. They were induced at least $30 \mathrm{~h}$ earlier when the plants were inoculated with $C$. heterostrophus than when inoculated with $C$. sublineolum (Fig. 4). In addition,

1 CTCCAAAACCACCAGCGGCAGCACCTTCAACCAGCGCAAGTTTAGTCGGCTCAGCTGATC

61 ACTGAAGCAATGGCCTCCGCCAACAGCTGGACCCTCGAGATCCCTTCGCCGGTGGCTGCG 120

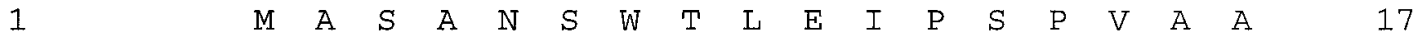

121 CGGCGCCTGTTCTGCGCCGCCGTGACACCCTGGCACCCAAGGTCAACTCCCAAGGTCAAC 180

$\begin{array}{lllllllllllllllllllllll}18 & R & R & L & F & C & A & A & V & T & P & W & H & P & R & S & T & P & K & V & N & & 37\end{array}$

181 TCCCACGTCGTCGCCAGCGCCCACCCCGTGGAGGACGACGGCGGCGTCGGCAGCGTCAGG 240

$\begin{array}{lllllllllllllllllllllll}38 & S & H & V & V & A & S & A & H & P & V & \text { E } & \text { D } & \text { D } & \text { G } & \text { G } & \text { V } & \text { G } & \text { S } & \text { V } & \text { R } & & 57\end{array}$

241 CAGTTCAACTTCACCTCATTCATGCCGTTCAGCTTCATGAAGGAGAGGCTCGATTTCCTG 300

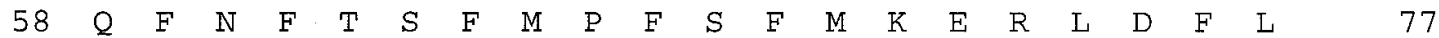

301 GACGTGGACAAGTGCGAGTGCAAGAACACGCTCGTCGAGGGCGGCAACATGCGACGTCGC 360

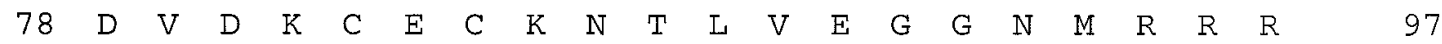

361 ATCGAGACCGCGGCGTCGCACATCAAGGTGGAGCCCGCGGCAGGCGGCGGCAGCGTGGTG 420

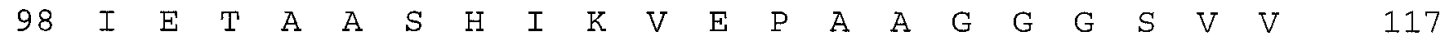

421 AAGGTGGAGTCGACCTACAAGCTGCTGCGGGGTGTGGACGCGAAGGACGAGGAAGCCAAG 480

$\begin{array}{lllllllllllllllllllllll}118 & K & V & E & S & T & Y & K & L & L & R & G & V & D & A & K & D & E & E & A & K & & 137\end{array}$

481 GCCAAGGAGGCACTCACCGCCATTTTCAAGGCTGCCGAGGCCTACCTCGTCGCCAACCCC 540

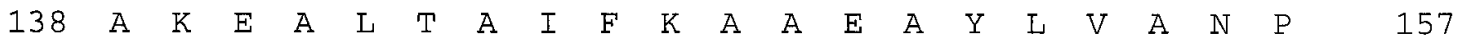

541 GACGCCTACAACTAAATCTGTGGATTGGGATGTGATTCCCCTTCCTGAAGTTCGGGTTTA 600

158 D A Y N * $\quad$ * $\quad *$ * 161

601 ATTTTCTTTTACTTTCCTGGAGTTTTCCTCTTTTTTTCATACTAAATAAAATAAGCTGCT 660

661 CTATGCGGTGTGTGGTGTATGGACGCAAATGTTATAAGTGGTTTGTGATGCTTTGTTTGT 720

721 GTGATATGTATAgTTGTATACCCTCATGgTTCTTTTCTATGTGGgAAgAAAAAGgGCAT 780

781 ACATAGATTATTCGAGGTACTTTGTTTTGGAATCCTATGCAAGTTTTGACTGTAAGAAAT 840

841 GTTTGCTCATTTCGAATAGATATTTGGAGACTTTTCATTCTGCTAAAAAAAAAAAAAAAA 900

901 AAAAAAA

907

Fig. 1. cDNA and predicted amino acid sequences of the sorghum PR-10 protein. The nucleotide and amino acid residues are numbered in the margins. The potential polyadenylation signal is underlined and putative termination codons are marked by asterisks. 
maximal levels of PR-10 and CHS transcripts accumulated over a short period of time $(24 \mathrm{~h})$ when the plants were inoculated with $C$. heterostrophus, compared with more than $48 \mathrm{~h}$ when the plants were inoculated with C. sublineolum (Fig. 4).

Another series of experiments was performed in which only the bottom half of the mesocotyls was inoculated with either $C$. heterostrophus or C. sublineolum, leaving the top half uninoculated. Under these conditions, only the bottom half (inoculated) of the mesocotyls synthesized the 3-deoxyanthocyanidin phytoalexins, which were visualized as red lesions on the plant surface (Nicholson et al. 1987). Northern blot analysis demonstrated that both PR-10 and CHS transcripts accumulated only in the inoculated (bottom) portion of the plants where the phytoalexin pigments were found, regardless of which fungus was used (Fig. 5). It should be noted that there was no basal expression of these genes in mesocotyls that were either uninoculated or mock inoculated with water containing Tween 20 (data not shown). Taken together, these results clearly suggest that expression of the PR-10 gene is not activated in a pathogenspecific or systemic manner, but is localized in the area of the phytoalexin response along with the activation of the CHS gene.

\section{Cytological studies of fungal development on inoculated mesocotyls.}

In an attempt to correlate the temporal expression of defenserelated genes in sorghum to different stages of the fungal infection process, morphological development of the fungi on inoculated plants was examined by light microscopy. C. heterostro-

Sorghum PR-10
Asparagus AoPR1
Potato pSTH-21
Parsley PR1-1
Pea pI49
Bean PR-10a
Ginseng RNase 2
Birch BetvI
Celery api g 1

Sorghum PR-10

Asparagus AoPR1

Potato pSTH-21

Parsley PR1-1

Pea pI49

Bean PR-10a

Ginseng RNase 2

Birch BetvI

Celery api g 1

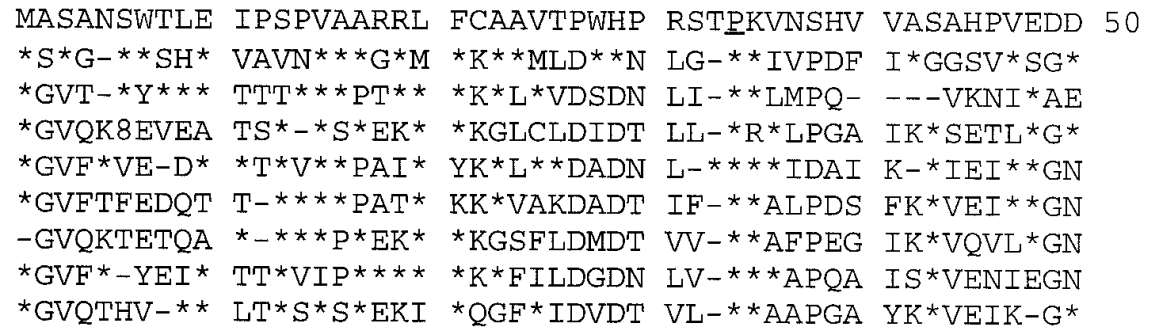

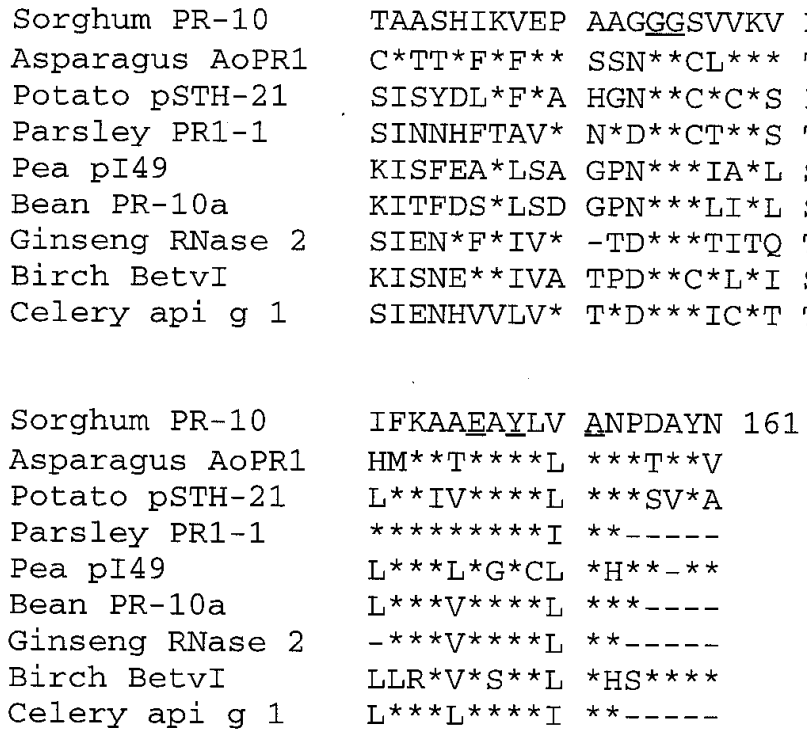

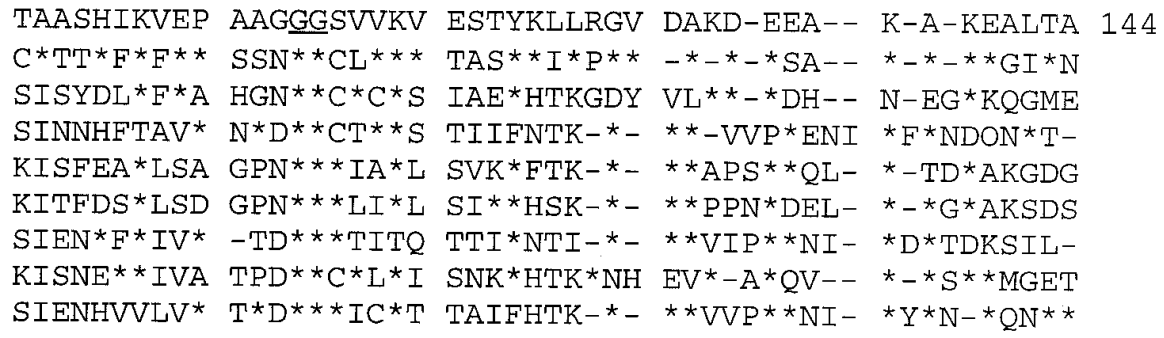

Fig. 2. Alignment of amino acid sequence of PR-10 and related proteins from different plants. The predicted sorghum PR-10 sequence is compared with the primary structures of the asparagus AoPR1 protein (Warner et al. 1992), the potato pSTH-21 (Matton and Brisson 1989), the parsley PR1-1 protein (Somssich et al. 1988), the pea disease response gene protein pI49 (Fristensky et al. 1988), the bean PR-10a protein (Walter et al. 1996), the birch pollen allergen BetvI (Breiteneder et al. 1989), the celery major allergen Api g 1 (Breiteneder et al. 1995), and the ginseng ribonuclease 2 (Moiseyev et al. 1997). Strictly conserved amino acid residues are underlined; dashes indicate gaps introduced for maximum alignment; asterisks replace identical residues. 
phus is the causal agent of southern corn leaf blight, but it is a nonpathogen of sorghum. We have previously shown that inoculation with $C$. heterostrophus caused rapid induction of phytoalexin synthesis in mesocotyl or leaf tissues (Nicholson et al. 1987, 1988; Lo et al. 1996). Conidia of C. heterostrophus germinated at both ends within $1 \mathrm{~h}$ after inoculation of the mesocotyls. By $4 \mathrm{~h}$ after inoculation, appressorial initials generally had formed at the tips of the germ tubes (Fig. 6A). Appressoria matured within $6 \mathrm{~h}$ of inoculation (Fig. 6B), and an appressorium was judged to have matured as indicated by the formation of a septum that delimits the appressorium from the germ tube (Weiergang et al. 1996a). The appressorium is the fungal infection structure from which penetration of host tissue originates. Within $9 \mathrm{~h}$ of inoculation, a mass of distorted hyphae was commonly found to emerge from mature appressoria (Fig. $6 \mathrm{C}$ ), suggesting failure of attempted penetrations. Extensive mycelial growth was later observed on the plant surface by $12 \mathrm{~h}$ after inoculation (Fig. 6D). During this time, the epidermal cells started to turn red, and red, pigmented bodies were found along the surface of the fungal mycelium, suggesting the accumulation of phytoalexin (Fig. 6D). Accumulation of phytoalexins became more apparent by $24 \mathrm{~h}$ after inoculation. Pigments were highly localized beneath the distorted hyphae growing on the plant surface (Fig. 6E). High levels of phytoalexin accumulation could also be observed in the plant cells in the vicinity of appressoria within $48 \mathrm{~h}$ of inoculation (Fig. 6F).

C. sublineolum is the causal agent of the anthracnose disease of sorghum. Conidia of $C$. sublineolum germinated with the formation of appressoria by $6 \mathrm{~h}$ after inoculation of mesocotyls (Fig. 7A). Melanization of appressoria occurred within $9 \mathrm{~h}$ of inoculation (Fig. 7B). Conidial germlings did not undergo further morphological changes until $36 \mathrm{~h}$ after inoculation when

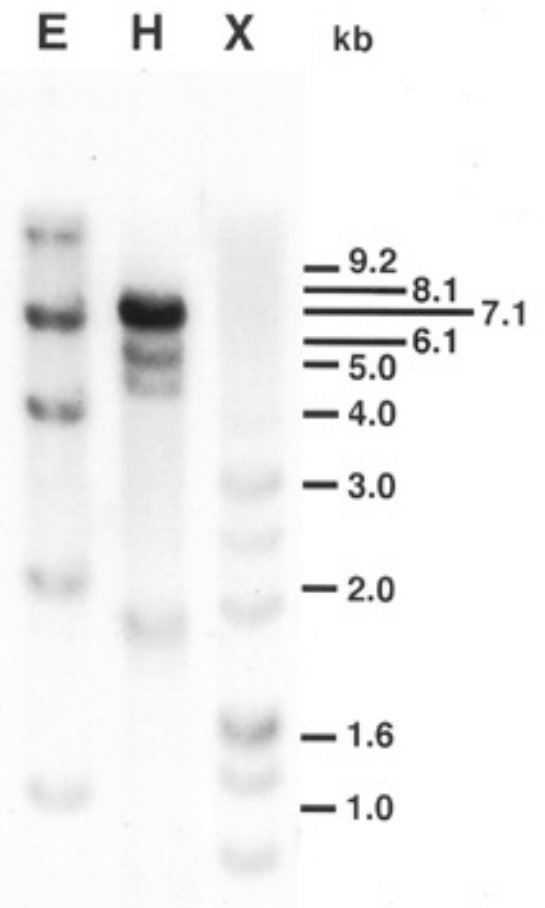

Fig. 3. Genomic Southern blot analysis of PR-10 genes in the sorghum genome. Total DNA isolated from sorghum mesocotyls $(10 \mu \mathrm{g})$ was digested with EcoRI (E), HindIII (H), or XbaI (X). Blot was hybridized with the sorghum PR-10 cDNA probe. DNA size marker positions are indicated. globose infection vesicles were formed within epidermal cells (Fig. 7C). Within $12 \mathrm{~h}$ of successful penetration (48 h after inoculation), primary hyphae grew from the infection vesicles and proliferated intracellularly (Fig. 7D). During this period, phytoalexin pigments were found to accumulate in the vicinity of the fungus. Both the infection vesicle and primary hyphae appeared distorted (Fig. 7D). By $72 \mathrm{~h}$ after inoculation, the phytoalexin pigments were distributed throughout infected cells and the fungal mycelium appeared collapsed and dead (Fig. 7E). Fungal development was therefore restricted in individual host cells and we concluded that the sorghum cultivar (DK18) used in the present study is resistant to C. sublineolum.

\section{Correlation of fungal morphological development with plant defense responses.}

A correlation exists between the morphological events of fungal development and the accumulation of PR-10 and CHS transcripts in inoculated sorghum mesocotyls. Defense-related responses were rapidly induced after inoculation with $C$. heterostrophus. Both PR-10 and CHS transcripts started to accumulate when the conidia had germinated and formed mature appressoria within $6 \mathrm{~h}$ of inoculation. Maximum levels of transcript accumulation occurred when there was extensive mycelial growth on the plant surface (12 h after inoculation) and localized accumulation of phytoalexin pigments followed (Figs. 4A and 6D-F). In contrast, PR-10 and CHS transcripts were not detected within $24 \mathrm{~h}$ of inoculation when sorghum mesocotyls were inoculated with $C$. sublineolum. (Fig. 4B). Appearance of the transcripts corresponded quite well to the formation of infection vesicles within epidermal cells (Fig. 7C). When the pathogen started to proliferate within the host cells (Fig. 7D), accumulation of PR-10 and CHS transcripts occurred rapidly and remained at high levels (Fig. 4B), followed by massive but localized accumulation of phytoalexin pigments (Fig. 7E).

\section{DISCUSSION}

In our search for genes up-regulated in sorghum responding to fungal inoculation, a cDNA clone coding for a transcript with a predicted gene product with high similarities to members of PR10 proteins was isolated. There is a widespread occurrence of PR-10 and related proteins across the plant kingdom. Evolutionary analysis of amino acid sequences revealed that PR-10 and related proteins of plants from the same taxonomic group displayed higher sequence homology to one another (Iturriaga et al. 1994; Moiseyev et al. 1997). Most of them were described in dicot families such as Leguminosae (alfalfa, bean, pea, and soybean), Solanaceae (potato), and Apiaceace (parsley and celery). Sorghum PR-10 protein, as expected, exhibits highest similarity (49.7\% identity) to a PR-10 protein (AoPR1) from asparagus, a monocot in the Liliaceae. AoPR1 was found to be distantly related to other dicot PR-10 and related proteins based on evolutionary sequence analysis (Iturriaga et al. 1994; Moiseyev et al. 1997). Therefore, sequence differences between the sorghum and asparagus proteins and other PR-10 proteins may reflect the differences between monocots and dicots (Warner et al. 1992).

The biological functions of PR-10 proteins are still unknown. Sequence analysis indicated that they contain no signal peptide or membrane-binding domains, suggesting that they are intracellular proteins located in the cytosol (Somssich et al. 1988; Matton and Brisson 1989; Warner et al. 1992). 
This is in contrast to the "classical" acidic PR proteins, which are generally extracellular (Bol et al. 1990). The rapid induction of PR-10 proteins after pathogen inoculation and elicitor treatment indicates that their expression could be part of the defense mechanisms. However, there are studies that suggested that these proteins are not a direct component of the first line of defense against potential pathogens. For example, washes of birch pollen with a high level of the allergen structurally similar to PR-10 proteins were not found to exhibit any antimicrobial activity (Swoboda et al. 1994). Transgenic potato plants overexpressing the PR-10 gene were reported to have no enhanced resistance to the pathogens Phytophthora infestans and potato virus X (Constabel et al. 1993).

There is, however, circumstantial evidence supporting the role for PR-10 proteins as ribonucleases. Recent evolutionary analysis revealed sequence similarities between two ginseng ribonucleases and members of the PR-10 and related proteins (Moiseyev et al. 1994, 1997). More recently, purified preparations of the natural and recombinant birch pollen allergen, BetvI, were shown to degrade plant RNA (Bufe et al. 1996; Swoboda et al. 1996). Walter et al. (1996) demonstrated spatio-temporal similarities between reported expression patterns of PR-10 genes and ribonuclease genes in bean. They postulated that PR10 proteins could function to degrade mRNA species induced during stress or pathogen attack in a selective manner. Such a mechanism would allow for the return to normal metabolic functioning of the plant. Consistent with this hypothesis, we have recently observed that accumulation of normal anthocyanins in inoculated sorghum mesocotyls resumed as soon as the synthesis of phytoalexins started to level off (Lo and Nicholson 1998). Following fungal inoculation of the plants, the normal anthocyanin synthesis pathway is immediately directed to the synthesis of 3-deoxyanthocyanidin phytoalexins.

Expression of a number of PR genes is systemically induced and is shown to be associated with systemic acquired resistance (Brederode et al. 1991; Ward et al. 1991). Accumulation of PR10 transcripts has always been localized in the regions of wounding or fungal inoculation and adjacent areas (Somssich et al. 1988; Schmelzer et al. 1989; Warner et al. 1992, 1993; this report). In contrast to the current study, Breda et al. (1996) demonstrated the accumulation of PR-10 transcripts in regions of the same alfalfa leaf either infiltrated or not infiltrated with Pseudomonas syringae pv. pisi. In situ hybridizations also indicated that the alfalfa PR-10 gene was expressed in vascular tissues

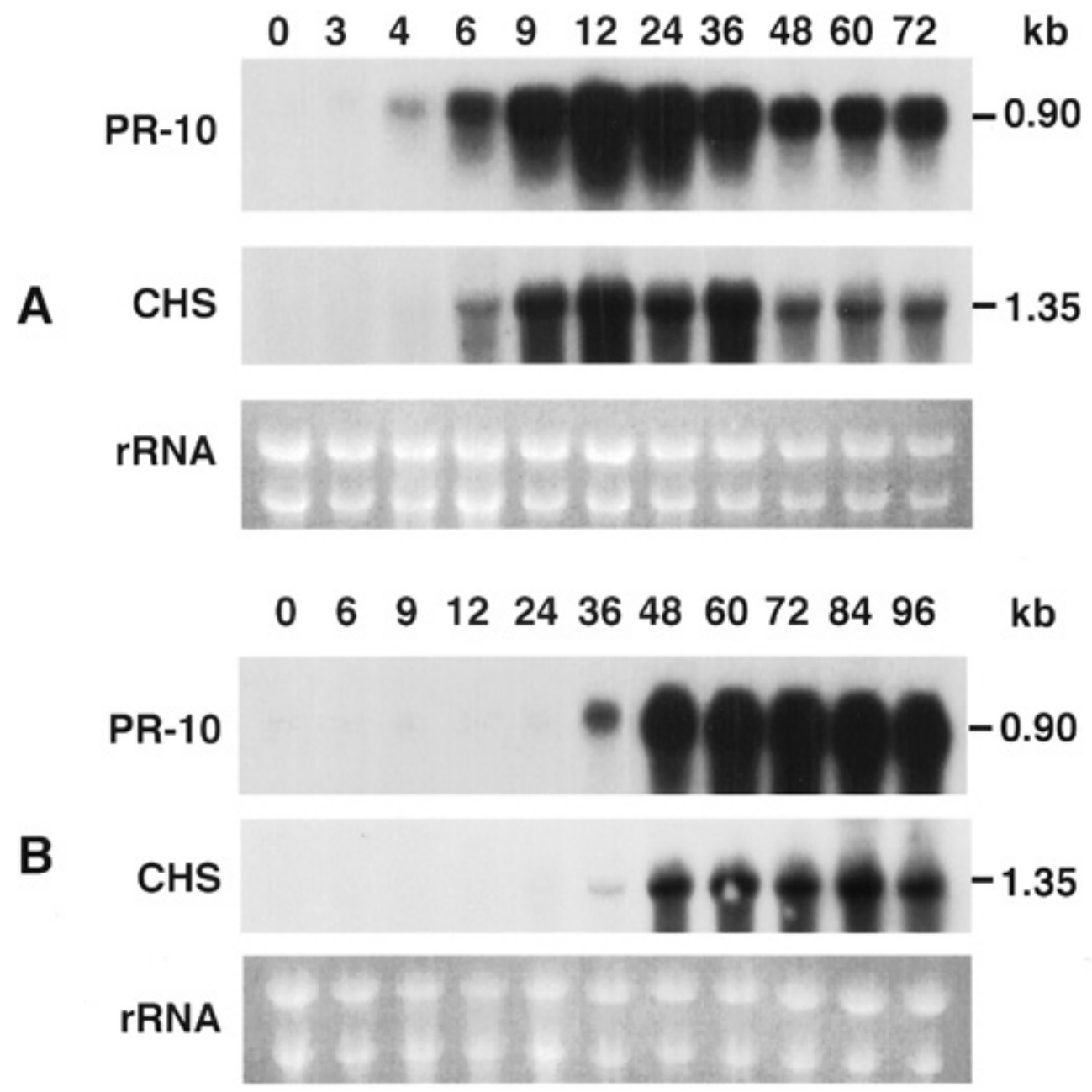

Fig. 4. Temporal expression of PR-10 and chalcone synthase (CHS) genes in sorghum mesocotyls inoculated with (A) Cochliobolus heterostrophus or (B) Colletotrichum sublineolum. Northern (RNA) blots are of total RNA $(10 \mu \mathrm{g})$ from mesocotyls taken at different time intervals, probed with the sorghum PR-10 cDNA clone or a CHS PCR (polymerase chain reaction) fragment (Cui et al. 1996). Numbers indicate hours after fungal inoculation. Equal loadings of RNA are shown by the rRNA in agarose gels stained with ethidium bromide. Sizes of the transcripts detected are also indicated. 
adjacent to and distant from the site of bacterial infiltration (Breda et al. 1996). On the other hand, PR-10 genes have also been demonstrated to be developmentally regulated. For example, there are reports on the constitutive expression of PR-10 genes in roots, dark-treated leaves or roots, and leaves undergoing senescence (Crowell et al. 1992; Warner et al. 1992; Breda et al. 1996; Walter et al. 1996). PR-10 proteins and promoterdriven activities were also detected in floral and vascular tissues (Warner et al. 1994; Constabel and Brisson 1995). Interestingly, the asparagus AoPR1 gene was specifically expressed in tissues accumulating products of the main branches of the phenylpropanoid pathway (Warner et al. 1994). In the present study, coordinate expression of genes encoding PR-10 protein and CHS in inoculated sorghum mesocotyls (Figs. 4 and 5) suggests that they are components of the fungal-inducible network triggered by similar signaling pathways. Promoter studies revealed that the asparagus AoPR1 gene contains sequence motifs similar to the $\mathrm{H}$ box sequences identified in the promoters of genes coding for enzymes of the phenylpropanoid pathway (Warner et al. 1994).

Our results also demonstrate that similar components of defense-related mechanisms (PR-10, CHS, and phytoalexins) are activated in sorghum mesocotyls following inoculation with either a nonpathogen ( $C$. heterostrophus) or a pathogen $(C$. sublineolum). Accumulation of phytoalexin pigments appears to be associated with the restriction of fungal development in the hosts (Figs. 6 and 7). The 3-deoxyanthocyanidin phytoalexins have been shown to exhibit in vitro toxicity toward both fungi. Germination of $C$. heterostrophus conidia was not affected whereas germ tube elongation of the fungus was inhibited by increasing concentrations of the 3-deoxyanthocyaindin

Bottom Top

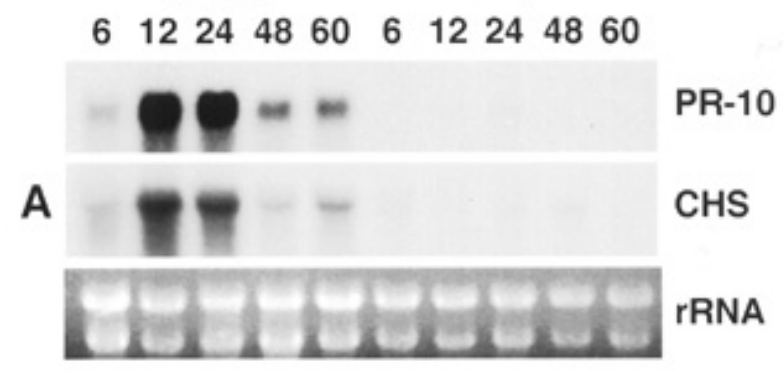

$48 \quad 60728496 \quad 4860728496$

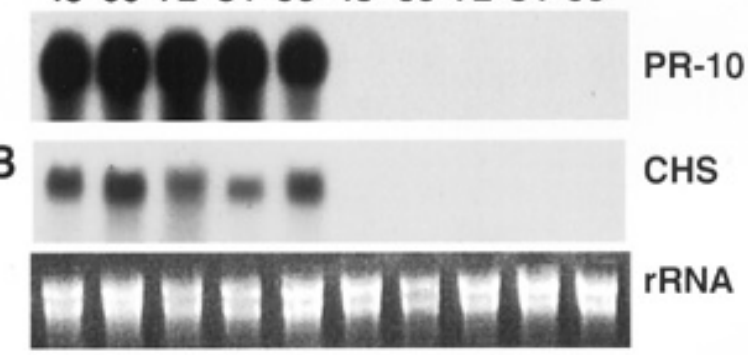

Fig. 5. Time course of accumulation of PR-10 and chalcone synthase (CHS) transcripts in sorghum mesocotyls following local inoculation with (A) Cochliobolus heterostrophus or (B) Colletotrichum sublineolum. Total RNA was extracted from the bottom half of mesocotyls inoculated with either fungus and the top half of the same mesocotyls that were not inoculated. Northern (RNA) blots were performed with $10 \mu \mathrm{g}$ of RNA in each lane and were hybridized with PR-10 and CHS probes as in Figure 4. Equal loadings of RNA are shown by the rRNA in agarose gels stained with ethidium bromide. compounds (Hipskind et al. 1990; Nicholson et al. 1987). With $C$. sublineolum, both germination and germ tube elongation were inhibited by increasing concentrations of the phytoalexins (Hipskind et al. 1990; Lo et al. 1996).

Two different kinds of resistance reactions are displayed by the sorghum cultivar (DK18) used in the present study, i.e., nonhost resistance to $C$. heterostrophus and host-specific resistance to $C$. sublineolum. There is a strong correlation of a rapid accumulation of PR-10, CHS transcripts, and phytoalexins with the resistance response to $C$. heterostrophus. Although corn and sorghum are closely related and have many pathogens in common, $C$. heterostrophus is nonpathogenic to sorghum. Cytological studies revealed no signs of successful penetration into the plant cells by the fungus (Fig. 6). It is possible that transcriptional activation of PR-10 and CHS genes and the accumulation of phytoalexins are triggered by some nonspecific structural or chemical features in C. heterostrophus or general stress associated with attempted invasion (Heath 1981; Johal et al. 1995). In the host resistant reaction to $C$. sublineolum, a delay in the activation of these defense-related responses was observed (Figs. 4 and $7 \mathrm{D}, \mathrm{E})$, suggesting that a different recognition event was involved for activation of defense response. Germination and appressorium formation of $C$. sublineolum were completed within $6 \mathrm{~h}$ of inoculation (Fig. 7). Both events were also completed in the same time frame by $C$. heterostrophus (Fig. 6). However, transcripts of PR-10 and CHS genes were not detectable until 36 $\mathrm{h}$ after inoculation when successful penetration by $C$. sublineolum was completed with the formation of infection vesicles. $C$. sublineolum has been classified as an intracellular hemibiotrophic pathogen with a biotrophic phase during some stages of pathogenesis (Wharton and Julian 1996). Biotrophic pathogens are believed to possess some unknown basic compatibility factors to evade the plant's "surveillance" system (Johal et al. 1995). Thus, $C$. sublineolum may suppress defense mechanisms that are triggered non-specifically by stress or general elicitors released during the attempted invasion process. However, special signals could be generated during the process and may be recognized by specific resistance gene products in the plant, leading to the subsequent activation of defense mechanisms. Such signals could be of pathogen or host origin, or a combination of both, resulting from the activities of avirulent gene products from the fungus (Clarke et al. 1992). Results from the current study present a unique monocot model for the elucidation of recognition and signaling events leading to the expression of resistance to $C$. heterostrophus and $C$. sublineolum.

\section{MATERIALS AND METHODS}

\section{Plant and fungal materials.}

Seeds of the sorghum (Sorghum bicolor (L.) Moench) cultivar DK18 (Dekalb Pfizer Genetics, Lubbock, TX) were planted in rolls of germination paper as described previously (Lo et al. 1996). The fungi used were Cochliobolus heterostrophus (Drechs.) Drechs., (anamorphic stage Bipolaris maydis (Nisikado \& Miyake) Shoemaker, culture Hmo-120, isolated in 1979 from maize in West Lafayette, IN), which is nonpathogenic to sorghum, and Colletotrichum sublineolum P. Henn, Kabát \& Bubák (Sheriff et al. 1995), isolate TX430BB (a gift from R. Frederiksen, Texas A\&M University, College Station), which causes the anthracnose disease of sorghum. C. heterostrophus and $C$. sublineolum were cultured on potato dextrose 
agar and oatmeal agar, respectively, under constant light $(60 \mu \mathrm{E}$. $\mathrm{s}^{-1} \cdot \mathrm{m}^{-2}$ ) at room temperature.

Four-day-old etiolated seedlings were inoculated with conidial suspensions of $C$. heterostrophus or $C$. sublineolum at
$5.5 \times 10^{4}$ or $1.0 \times 10^{6}$ conidia $\mathrm{ml}^{-1}$, respectively. Tween 20 was used as a wetting agent $\left(100 \mu 1100 \mathrm{ml}^{-1}\right)$ in the inoculum suspension. The inoculated plants were incubated at $100 \%$ relative humidity at room temperature under constant light. To
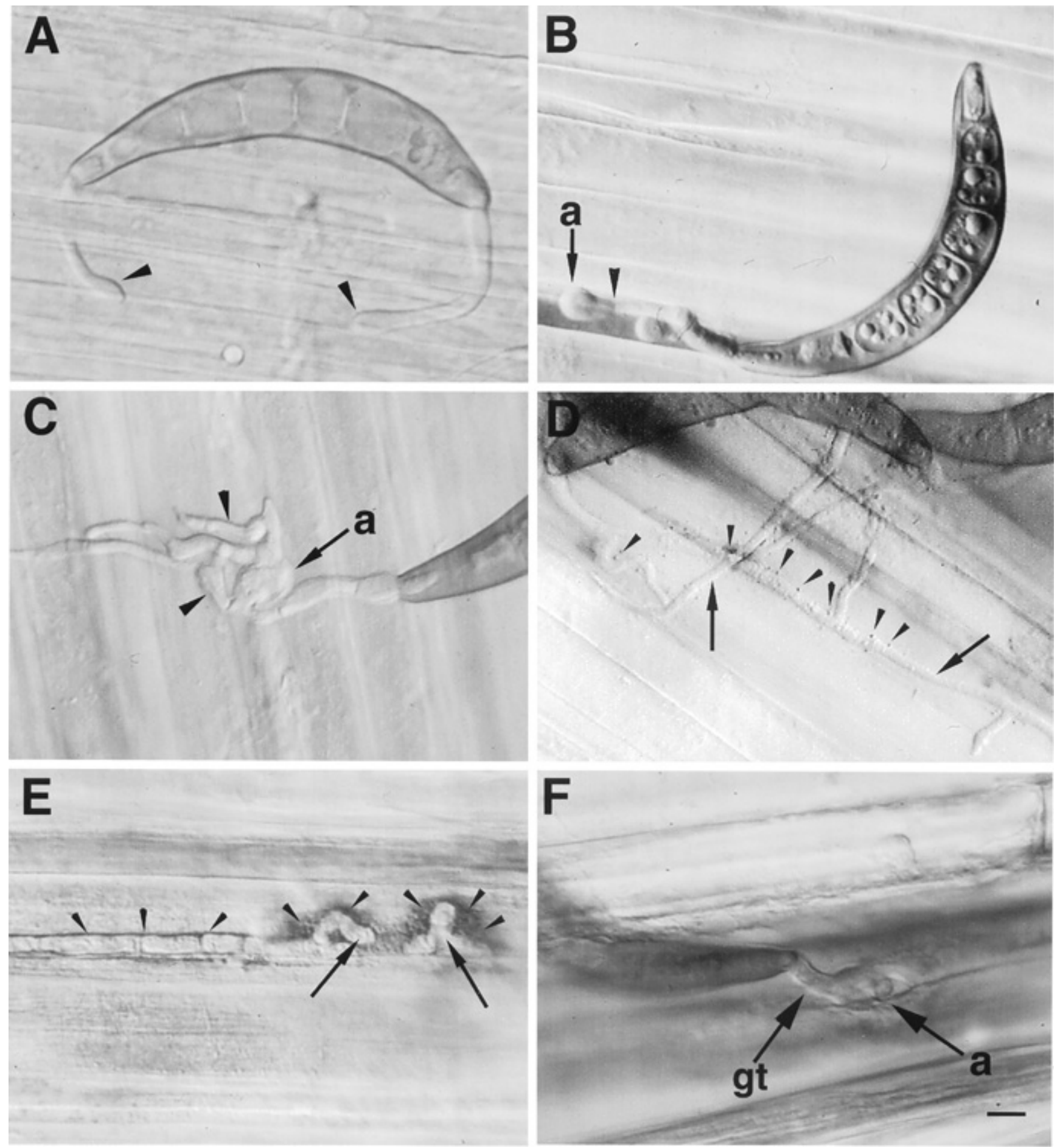

Fig. 6. Infection process of Cochliobolus heterostrophus on sorghum mesocotyls examined by light microscopy. A, $4 \mathrm{~h}$ after inoculation, germination of a conidium with the formation of appressorial initials (arrows) was observed. B, $6 \mathrm{~h}$ after inoculation, maturation of an appressorium (a) was indicated by the presence of a septum (arrow) delimiting the appressorium from the germ tube. $\mathbf{C}, 9 \mathrm{~h}$ after inoculation, failure of attempted penetration by the fungus as indicated by the presence of distorted hyphae (arrows) that emerged from the appressorium (a). D, $12 \mathrm{~h}$ after inoculation, mycelial growth was found on the plant surface (arrows). At this time cells of the underlying epidermis had become pigmented, indicating the accumulation of phytoalexins. Small pigmented bodies (arrowheads) were present on the surface of the hyphae. E, $24 \mathrm{~h}$ after inoculation, superficial hyphae appeared thickened and distorted (arrows). Hyphae were surrounded by intensely pigmented materials, indicating the presence of phytoalexins (arrowheads). F, $48 \mathrm{~h}$ after inoculation, appressorium (a), germ tube (gt), and the body of the conidium were stained with the pigmented phytoalexins. Bar represents $10 \mu \mathrm{m}$. 
investigate possible effects of fungal stimuli on systemic gene expression, etiolated seedlings were partially protected by plastic film on the top and were inoculated with either fungus on the bottom.
cDNA library construction and screening, plaque rescue, and DNA sequencing.

Total RNA and poly (A) ${ }^{+}$RNA were isolated from sorghum seedlings $24 \mathrm{~h}$ after inoculation with $C$. sublineolum as de-
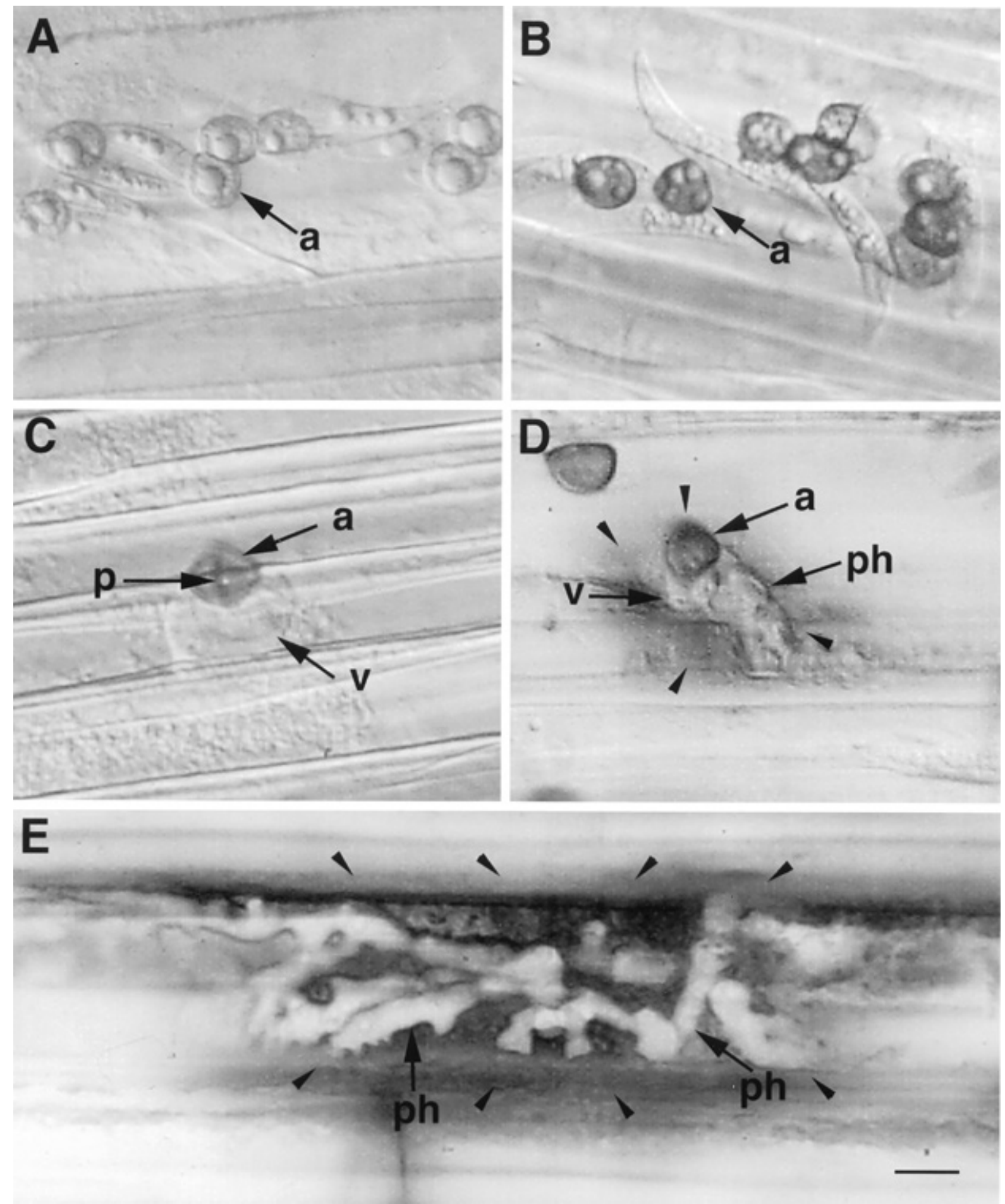

Fig. 7. Infection process of Colletotrichum sublineolum on sorghum mesocotyls examined by light microscopy. A, $6 \mathrm{~h}$ after inoculation, germination of conidia with the formation of appressoria (a) was observed. B, $9 \mathrm{~h}$ after inoculation, melanization of appressoria (a) occurred as indicated by their dark pigmentation. $\mathbf{C}, 36 \mathrm{~h}$ after inoculation, penetration of the fungus into host tissue had been completed with the formation of an infection vesicle (v). A penetration pore (p) is present in the appressorium (a). D, $48 \mathrm{~h}$ after inoculation, a primary hypha (ph) had grown out from the infection vesicle (v). The area (arrowheads) surrounding the appressorium (a), infection vesicle (v), and primary hypha (ph) was intensely pigmented by the phytoalexin. E, $72 \mathrm{~h}$ after inoculation, distorted primary hyphae (ph) are surrounded by an area with intensely pigmented phytoalexins (arrowheads) in an infected cell. Bar represents $10 \mu \mathrm{m}$. 
scribed previously (Hipskind et al. 1996b). The poly (A) ${ }^{+}$ RNA then served as the template for cDNA synthesis and construction of a lambda ZAP-cDNA library according to the manufacturer's instructions (Stratagene, La Jolla, CA). The resulting library contained $1.2 \times 10^{6} \mathrm{PFU}$. Ten thousand plaques were plated out at low density and duplicated filters (BA-S 85 Optitran membranes; Schleicher and Schuell, Keene, $\mathrm{NH}$ ) were lifted from the plates. One set of filters was probed with ${ }^{32} \mathrm{P}$-labeled first-strand cDNA synthesized from the same poly $(\mathrm{A})^{+}$RNA used to construct the library and the other set was probed with ${ }^{32} \mathrm{P}$-labeled first-strand cDNA synthesized from uninoculated mesocotyls. Hybridizations were performed essentially as described (Hipskind et al. 1996b). Autoradiography was carried out overnight with X-ray films (XOMAT AR; Kodak, New Haven, CT) with intensifying screens at $-70^{\circ} \mathrm{C}$. Differentially hybridizing plaques were isolated and taken through two more rounds of differential hybridization.

The phagemids (pBluescript $\mathrm{SK}^{-}$) of the positive clones were rescued from phages following the in vivo excision protocol recommended by the manufacturer (Stratagene). Both strands of the recombinant phagemids were sequenced at the Purdue Center for DNA Sequencing (West Lafayette, IN). These sequence data and subsequent predicted translation from the first ATG were used in a computer-aided on-line search of nucleotide and protein data bases through the BLAST network service (National Center for Biotechnology Information, Bethesda, MA; Altschul et al. 1990). Amino acid sequence alignment and analysis were achieved with the use of the MacDNASIS software program (Hitachi Software, San Bruno, CA).

\section{DNA isolation and Southern blotting.}

DNA was isolated from freeze-dried mesocotyl tissue of sorghum cultivar DK18 according to the CTAB nucleic acid procedure (Rogers and Bendich 1988). DNA was treated with RNase and 10- $\mu$ g samples were digested to completion with either EcoRI, HindIII, or XbaI. The digested DNA was separated by electrophoresis on a $0.8 \%$ agarose gel, depurinated, denatured, and blotted in $10 \times \mathrm{SSC}(1 \times \mathrm{SSC}$ is $0.15 \mathrm{M} \mathrm{NaCl}$ plus $0.015 \mathrm{M}$ sodium citrate) onto a nylon membrane (Zeta-Probe GT; BioRad, Hercules, CA) by downward capillary transfer (Zhou et al. 1994) for at least $16 \mathrm{~h}$ then covalently cross-linked to the membrane with a UV cross-linker (Fisher Scientific, Pittsburgh, PA).

\section{RNA isolation and Northern blotting.}

Total RNA was isolated from liquid nitrogen-frozen mesocotyl tissues by the procedure of Hipskind et al. (1996a). Aliquots of $10 \mu \mathrm{g}$ were denatured and fractionated on a formaldehyde-1.0\% agarose gel in MOPS (morpholinepropanesulfonic acid) buffer. Equal loading of RNA on gels was checked by means of ethidium bromide-stained gels. Following electrophoresis, RNA was transferred by downward capillary action (Zhou et al. 1994) to nylon membranes (ZetaProbe GT; Bio-Rad) for at least $16 \mathrm{~h}$, then covalently crosslinked to the membrane with a UV cross-linker. The sizes of mRNA species detected were estimated by use of a 0.28 to 6.6 kb RNA ladder (Sigma, St. Louis, MO).

\section{Southern and Northern hybridizations.}

Southern and Northern blots were pre-hybridized for $15 \mathrm{~min}$ at $65^{\circ} \mathrm{C}$ in $0.5 \mathrm{M}$ sodium phosphate buffer ( $\left.\mathrm{pH} 7.0\right)$ containing $7 \%$ (wt/vol) SDS (sodium dodecyl sulfate). Membranes were then hybridized for $18 \mathrm{~h}$ in the same buffer with the addition of different denatured ${ }^{32} \mathrm{P}$-labeled probes. An insert of approximately $800 \mathrm{bp}$ was released from the pBluescript SK(-) phagemid containing the PR-10 cDNA by XhoI and was used as a probe for Southern and Northern hybridizations. In addition, a 620-bp CHS PCR (polymerase chain reaction) fragment (Cui et al. 1996) was used as a probe for Northern hybridization. Radioactive DNA probes were prepared by random primer labeling with a Deca-Prime labeling kit (Ambion, Austin, TX). The hybridized membranes were washed three times in $0.5 \times \mathrm{SSC}, 0.5 \% \mathrm{SDS}$ at $65^{\circ} \mathrm{C}$. The membranes were then exposed to X-ray films (X OMAT-AR; Kodak) with intensifying screens at $-70^{\circ} \mathrm{C}$.

\section{Light microscopy.}

Morphological development of the fungi $C$. heterostrophus and $C$. sublineolum on the mesocotyl tissue was studied by light microscopy at indicated time intervals (Figs. 6 and 7). Strips of epidermal tissue were removed from inoculated mesocotyls, mounted in distilled water, and examined with bright field and/or Nomarski interference contrast optics.

\section{ACKNOWLEDGMENTS}

This research was supported in part by grant no. MCB-9603439 to R. L. N. from the National Science Foundation. This is journal article no. 15707 of the Purdue University Agricultural Experiment Station. We also thank C. Magill (Texas A\&M University, College Station) for the CHS PCR clone.

\section{LITERATURE CITED}

Altschul, S. F., Gish, W., Miller, W., Myers, E. W., and Lipman, D. J. 1990. Basic local alignment search tool. J. Mol. Biol. 215:403-410.

Bohlmann, H., Clausen, S., Behnke, S., Giese, H., Hiller, C., ReimannPhilipp, U., Schrader, G., Barholt, V., and Apel, K. 1988. Leafspecific thionins of barley - a novel class of cell wall proteins toxic to plant pathogenic fungi and possibly involved in the defense mechanism of plants. EMBO J. 7:1559-1565.

Bol, J., Linthorst, H. J. M., and Cornelissen, B. J. C. 1990. Plant pathogenesis-related proteins induced by virus infection. Annu. Rev. Phytopathol. 28:13-138

Breda, C., Sallaud, C., El-Turk, J., Buffard, D., de Kozak, I., Esnault, R., and Kondorosi, A. 1996. Defense reaction in Medicago sativa: A gene encoding a class $10 \mathrm{PR}$ protein is expressed in vascular bundles. Mol. Plant-Microbe Interact. 9:713-719.

Brederode, F. T., Linthorst, J. M., and Bol, J. F. 1991. Differential induction of acquired resistance and PR gene expression in tobacco by virus infection, ethephon treatment, UV light and wounding. Plant Mol. Biol. 17:1117-1125.

Breiteneder, H., Hoffmann-Sommergruber, K., O’Riordain, G. O., Susani, M., Ahorn, H., Ebner, C., Kraft, D., and Scheiner, O. 1995. Molecular characterization of Api g 1, the major allergen of celery (Apium graveolens), and its immunological and structural relationships to a group of 17-kDa tree pollen allergens. Eur. J. Biochem. 233:484:489.

Breiteneder, H., Pettenburger, K., Bito, A., Valenta, R., Kraft, D., Rumpold, H., Schneider, O., and Breitenbach, M. 1989. The gene coding for the major birch pollen allergen BetvI is highly homologous to a pea disease resistance response gene. EMBO J. 8:1935-1938.

Bufe, A., Spangfort, M. D., Kahlert, H., Schlaak, M., and Becker, W.-M. 1996. The major birch pollen allergen, Bet v 1, shows ribonuclease activity. Planta 199:413-415.

Clarke, H. R. G., Leigh, J. A., and Douglas, C. J. 1992. Molecular signals in the interactions between plants and microbes. Cell 71:191-199.

Collinge, D. B., Kragh, K. M., Mikkelsen, J. D. Nielsen, K. K., Rasmussen, U., and Vad, K. 1993. Plant chitinases. Plant J. 3:21-40.

Constabel, C. P., Bertrand, C., and Brisson, N. 1993. Transgenic potato plants overexpressing the pathogenesis-related STH-2 gene show unaltered susceptibility to Phytophthora infestans and potato virus X. Plant Mol. Biol. 22:775-782.

Constabel, C. P., and Brisson, N. 1995. Stigma- and vascular-specific expression of the PR-10a gene of potato: A novel pattern of expression of a pathogenesis-related gene. Mol. Plant-Microbe Interact. 8:104-113. 
Crowell, D. N., John, M. E., Russel, D., and Amasino, R. M. 1992. Characterization of a stress-induced, developmentally regulated gene family from soybean. Plant Mol. Biol. 18:459-466.

Cui, Y., Magill, J., Frederiksen, R., and Magill, C. 1996. Chalcone synthase and phenylalanine ammonia-lyase mRNA levels following exposure of sorghum seedlings to three fungal pathogens. Physiol. Mol. Plant Pathol. 49:187-199.

Cutt, J. R., and Klessig, D. F. 1992. Pathogenesis-related proteins. Pages 209-243 in: Genes Involved in Plant Defense. T. Boller and F. Meins, Jr., eds. Springer, Vienna.

Fristensky, B., Horovitz, D., and Hadwiger, L. A. 1988. cDNA sequences for pea disease response genes. Plant Mol. Biol. 11:713-715.

Gajhede, M., Osmark, P., Poulsen, F. M., Ipsen, H., Larsen, J. N., van Neerven, R. J. J., and Schou, C. 1996. X-ray and NMR structure of Bet v 1, the origin of birch pollen allergy. Nature Struct. Biol. 3:1040-1045.

Heath, M. C. 1981. A generalized concept of host-parasite specificity. Phytopathology 71:1121-1123.

Hipskind, J. D., Goldsbrough, P. B., Urmeev, F., and Nicholson, R. L. 1996a. Synthesis of 3-deoxyanthocyanidin phytoalexins in sorghum does not occur via the same pathway as 3-hydroxylated anthocyanidins and phlobaphenes. Maydica 41:155-166.

Hipskind, J. D., Hanau, R., Leite, B., and Nicholson, R. L. 1990. Phytoalexin accumulation in sorghum: Identification of an apigeninidin acyl ester. Physiol. Mol. Plant Pathol. 36:381-396.

Hipskind, J. D., Nicholson, R. L., and Goldsbrough, P. B. 1996b. Isolation of a cDNA encoding a novel leucine-rich repeat motif from Sorghum bicolor inoculated with fungi. Mol. Plant-Microbe Interact. 9:819-825.

Iturriaga, E. A., Leech, M. J., Barratt, D. H. P., and Wang, T. L. 1994. Two ABA-responsive proteins from pea (Pisum sativum L.) are closely related to intracellular pathogenesis-related proteins. Plant Mol. Biol. 24:235-240.

Johal, G. S., Gray, J., Gruis, D., and Briggs, S. P. 1995. Convergent insights into mechanisms determining disease and resistance response in plant-fungal interactions. Can. J. Bot. 73 (Suppl. 1):S468-S474.

Kauffmann, S., Legrand, M., Geoffroy, P., and Fritig, B. 1987. Biological function of "pathogenesis-related" proteins: Four PR proteins of tobacco have 1,3- $\beta$-glucanase activity. EMBO J. 6:3209-3212.

Keen, N. T. 1992. Gene-for-gene complementarity in plant-pathogen interactions. Annu. Rev. Genet. 24:447-463.

Lo, S.-C., and Nicholson, R. L. 1998. Reduction of light-induced anthocyanin accumulation in inoculated sorghum mesocotyls. Implication for a compensatory role in the defense response. Plant Physiol. 116:979-989.

Lo, S.-C., Weiergang, I., Bonham, C., Hipskind, J., Wood, K., and Nicholson, R. L. 1996. Phytoalexin accumulation in sorghum: Identification of a methyl ether of luteolinidin. Physiol. Mol. Plant Pathol. 49:21-31.

Lue, W. L., Kuhn, D., and Nicholson, R. L. 1989. Chalcone synthase activity in sorghum mesocotyls inoculated with Colletotrichum graminicola. Physiol. Mol. Plant Pathol. 35:413-422.

Matton, D. P., and Brisson, N. 1989. Cloning, expression, and sequence conservation of pathogenesis-related gene transcripts of potato. Mol. Plant-Microbe Interact. 2:325-331.

Moiseyev, G. P., Beintema, J. J., Fedoreyeva, L. I., and Yakovlev, G. I. 1994. High sequence similarity between a ribonuclease from ginseng calluses and fungus-elicited proteins from parsley indicates that intracellular pathogenesis-related proteins are ribonucleases. Planta 193:470-472.

Moiseyev, G. P., Fedoreyeva, L. I., Zhuravlev, Y. N., Yasnetskaya, E., Jekel, P. A., and Beintema, J. J. 1997. Primary structures of two ribonucleases from ginseng calluses. New member of the PR-10 family of intracellular pathogenesis-related plant proteins. FEBS Lett. 402:207-210.

Nicholson, R. L., Jamil, F. F., Synder, B. A., Lue, W. L., and Hipskind, J. 1988. Phytoalexin synthesis in the juvenile sorghum leaf. Physiol. Mol. Plant Pathol. 33:271-278.

Nicholson, R. L., Kollipara, S. S., Vincent, J. R., Lyons, P. C., and Cadena-Gomez, G. 1987. Phytoalexin synthesis by the sorghum mesocotyl in response to infection by pathogenic and nonpathogenic fungi. Proc. Nat. Acad. Sci. USA 84:5520-5524.

Pautot, V., Holzer, F. M., and Walling, L. L. 1991. Differential expression of tomato proteinase inhibitor I and II genes during bacterial pathogen invasion and wounding. Mol. Plant-Microbe Interact. 4:284-292.

Ponstein, A. S., Bres-Vloemans, S. A., Sela-Buurlage, M. B., van den Elzen, P. J. M., Melchers, L. S., and Cornelissen, B. J. C. 1994. A novel pathogen- and wound-inducible tobacco (Nicotiana tabacum) protein with antifungal activity. Plant Physiol. 104:109-118.

Riggleman, R. C., Fristensky, B., and Hadwiger L. A. 1985. The disease resistance response in pea is associated with increased levels of specific mRNAs. Plant Mol. Biol. 4:81-86.

Roby, D., and Esquerre-Tugaye, M.-T. 1987. Purification and some properties of chitinases from melon plants infected by Colletotrichum lagenarium. Carbohydr. Res. 165:93-104.

Rogers, S. O., and Bendich, A. J. 1988. Extraction of DNA from plant tissue. Pages A6:1-10 in: Plant Molecular Biology Manual. S. B. Gelvin and R. A. Schilperoort, eds. Kluwer Academic Publishers, Dordrecht, The Netherlands.

Schmelzer, E., Kruger-Lebus, S., and Hahlbrock, K. 1989. Temporal and spatial patterns of gene expression around sites of attempted fungal infection in parsley leaves. Plant Cell 1:993-1001.

Sheriff, C., Whelan, M. J., Arnold, G. M., and Bailey, J. A. 1995. rDNA sequence analysis confirms the distinction between Colletotrichum graminicola and C. sublineolum. Mycol. Res. 99:475-478.

Somssich, I. E., Schmelzer, E., Kawalleck, P., and Hahlbrock, K. 1988. Gene structure and in situ transcript localization of pathogenesisrelated protein 1 in parsley. Mol. Gen. Genet. 213:93-98.

Stintzi, A., Heitz, T., Prasad, V., Wiedemann-Merdinoglu, S., Kauffmann, S., Geoffroy, P., Legrand, M., and Fritig, B. 1993. Plant pathogenesis-related proteins and their role in defense against pathogens. Biochimie 75:687-706.

Swoboda, I., Hoffmann-Sommergrube, K., O'Riordain, G., Scheiner, O., Heberle-Bors, E., and Vicente, O. 1996. Bet v 1 proteins, the major birch pollen allergens and members of a family of conserved pathogenesis-related proteins, show ribonuclease activity in vitro. Physiol. Plant. 96:433-438.

Swoboda, I., Scheiner, O., Kraft, D., Breitenbach, M., Heberle-Bors, E., and Vicente, O. 1994. A birch gene family encoding pollen allergens and pathogenesis-related proteins. Biochim. Biophys. Acta 1219:457-464

van Loon, L. C. 1985. Pathogenesis-related proteins. Plant Mol. Biol. 4: 111-116.

van Loon, L. C., Pierpoint, W. S., Boller, T., and Conejero, V. 1994. Recommendations for naming plant pathogenesis-related proteins. Plant Mol. Biol. Rep. 12:254-264.

van Loon, L. C., and van Kammen, A. 1970. Polyacrylamide disc electrophoresis of the soluble leaf proteins from Nicotiana tabacum var. 'Samsun' and 'Samsun NN' II. Changes in protein constitution after infection with tobacco mosaic virus. Virology 40:199-211.

Walter, M. H., Liu, J.-W., Grand, C., Lamb, C. J., and Hess, D. 1990. Bean pathogenesis-related (PR) proteins deduced from elicitor-induced transcripts are members of a ubiquitous new class of conserved PR proteins including pollen allergens. Mol. Gen. Genet. 222:353-360.

Walter, M. H., Liu, J.-W., Wunn, J., and Hess, D. 1996. Bean ribonuclease-like pathogenesis-related protein genes (Ypr10) display complex patterns of developmental, dark-induced and exogenous-stimulusdependent expression. Eur. J. Biochem. 239:281-293.

Ward, E. R., Uknes, S. J., Williams, S. C., Dincher, S. S., Wiederhod, D. L., Alexander, D. C., Ahl-Goy, P., Metraux, J.-P., and Ryals, J. A. 1991. Coordinate gene activity in response to agents that induce systemic acquired resistance. Plant Cell 3:1085-1094.

Warner, S., Gill, A., and Draper, J. 1994. The developmental expression of the asparagus intracellular PR protein (AoPR1) gene correlates with sites of phenylpropanoid biosynthesis. Plant J. 6:31-43.

Warner, S., Scott, R., and Draper, J. 1992. Characterization of a woundinduced transcript from the monocot asparagus that shares similarity with a class of intracellular pathogenesis-related (PR) proteins. Plant Mol. Biol. 19:555-561

Warner, S., Scott, R., and Draper, J. 1993. Isolation of an asparagus intracellular PR gene (AoPR1) wound-responsive promoter by the inverse polymerase chain reaction and its characterization in transgenic tobacco. Plant J. 3:191-201

Weiergang, I., Dunkle, D., Wood, K. V., and Nicholson, R. L. 1996a Morphogenic regulation of pathotoxin synthesis in Cochliobolus carbonum. Fungal Genet. Biol. 20:74-78.

Weiergang, I., Hipskind, J. D., and Nicholson, R. L. 1996b. Synthesis of 3-deoxyanthocyanidin phytoalexins in sorghum occurs independent of light. Physiol. Mol. Plant Pathol. 49:377-388.

Wharton, P. S., and Julian, A. M. 1996. A cytological study of compatible and incompatible interactions between Sorghum bicolor and Colletotrichum sublineolum. New Phytol. 134:25-34.

Zhou, M. Y., Yue, D., Gomez-Sanchez, P., and Gomez-Sanchez, C. E. 1994. Improved downward capillary transfer for blotting of DNA and RNA. Biotechniques 16:58-59. 